Inequalities and consequences of new convolutions for the fractional Fourier transform with Hermite weights

P. K. Anh, L. P. Castro, P. T. Thao, and N. M. Tuan

Citation: 1798, 020006 (2017); doi: 10.1063/1.4972598

View online: http://dx.doi.org/10.1063/1.4972598

View Table of Contents: http://aip.scitation.org/toc/apc/1798/1

Published by the American Institute of Physics 


\title{
Inequalities and Consequences of New Convolutions for the Fractional Fourier Transform with Hermite Weights
}

\author{
P.K. Anh ${ }^{1, b)}$, L.P. Castro ${ }^{2, a)}$, P.T. Thao ${ }^{3, c)}$ and N.M. Tuan ${ }^{4, d)}$ \\ ${ }^{1}$ Department of Mathematics, College of Science, Vietnam National University, Hanoi, Vietnam. \\ ${ }^{2}$ CIDMA - Center for Research and Development in Mathematics and Applications, Department of Mathematics, \\ University of Aveiro, 3810-193 Aveiro, Portugal. \\ ${ }^{3}$ Department of Mathematics, Hanoi Architectural University, Hanoi, Vietnam. \\ ${ }^{4}$ Department of Mathematics, College of Education, Vietnam National University, Hanoi, Vietnam.

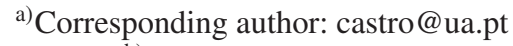 \\ b) anhpk@vnu.edu.vn \\ ${ }^{\text {c) }}$ phamthao.hau@gmail.com \\ d)nguyentuan@vnu.edu.vn
}

\begin{abstract}
This paper presents new convolutions for the fractional Fourier transform which are somehow associated with the Hermite functions. Consequent inequalities and properties are derived for these convolutions, among which we emphasize two new types of Young's convolution inequalities. The results guarantee a general framework where the present convolutions are well-defined, allowing larger possibilities than the known ones for other convolutions. Furthermore, we exemplify the use of our convolutions by providing explicit solutions of some classes of integral equations which appear in engineering problems.
\end{abstract}

\section{INTRODUCTION}

The roots of the fractional Fourier transform (FrFT) appeared long time ago, around 1929, mostly associated with the works of N. Wiener [34], H. Weyl, E.U. Condon [13], and H. Kober [21]. At a first period after birth, the FrFT was not immediately widely used in diverse subareas of knowledge, becoming even somehow forgotten (or, at least, not extensively used). After the works of Namias and McBride [22, 24], there was a great revival of pure and applied studies on the FrFT, mainly from the nineties of $20^{\text {th }}$ Century to the present. In part, this occurred due to the emergence, in that period, of several subareas where the application of the FrFT was recognized to be quite extensive and exhibiting great potential. This was the case in quantum mechanics, optics, signal processing, radar, watermarking, pattern recognition, cryptography, wavelet transforms and neural networks $[1,2,5,15,20,22,23,24,25,26,27,28,30,31,33,35]$.

In the recent past, we can recognize many efforts, explicit or implicit, to construct new convolutions for the FrFT. However, none of them can be seen as an appropriate generalization of the classical result for the Fourier transform. Namely, the papers $[3,4,14,31,33,35]$ introduced convolution formulas which are well-defined in some function spaces - mainly in $L^{1}(\mathbb{R})$ and in the Wiener algebra. Those convolutions are very interesting, and applicable to both theoretical and practical problems, since each one of them incorporate a new version of a suitable convolution theorem for the Fourier transform. However, due to the global nature of the FrFT, convolution and product theorems for the FrFT have not yet been intensively studied and obtained. In particular, there is no equal diversity of convolutions for the FrFT in comparison with those existent for the Fourier transform. Also, its corresponding Wiener algebra has not been addressed yet.

Motivated by this situation, this paper is devoted to propose new convolutions for the FrFT which are related with the Hermite functions. The new convolutions will imply several inequalities and consequences in a very global way. The paper is divided into four sections and organized as follows. In the next section, we provide three new convolution theorems which contain infinitely many new convolutions, and we prove their fundamental properties. The third section starts with a comparison and comprehensive analysis on the convolution and product theorems of the last six papers cited above. In special, this will lead us to Young's type inequalities. In particular, Theorem 4 indicates

ICNPAA 2016 World Congress

AIP Conf. Proc. 1798, 020006-1-020006-10; doi: 10.1063/1.4972598

Published by AIP Publishing. 978-0-7354-1464-8/\$30.00 
that the convolutions here proposed are well-defined in more flexible and better circumstances than the frameworks of the previously known ones. In the last section, by using the new convolutions, we investigate the solvability of some classes of convolution integral equations (which culminates with the derivation of explicit solution formulas). One of the types of integral equations under investigation (cf. (16)) has a universal Hermite kernel, having therefore a great potential of applicability to other sciences $[8,9,10,11,12,16,17]$.

\section{NEW CONVOLUTIONS}

In what follows, for the two-valued square root function $w=\sqrt{z}(z \in \mathbb{C})$ we choose the continuous branch taking $\sqrt{1}=1$. For a given angle $\alpha \in \mathbb{R}$, we put $a(\alpha):=\cot (\alpha) / 2, b(\alpha):=\sec (\alpha)$ and $c(\alpha):=\sqrt{1-i \cot (\alpha)}$. The FrFT with angle $\alpha$ is defined by

$$
\mathcal{F}_{\alpha}[f](p):=\frac{c(\alpha) e^{i a(\alpha) p^{2}}}{\sqrt{2 \pi}} \int_{-\infty}^{\infty} e^{i a(\alpha)\left(x^{2}-2 b(\alpha) x p\right)} f(x) d x,
$$

where we consider $\alpha \neq k \pi$ for every $k \in \mathbb{Z}$, for simplicity. We recall that only in the case $\alpha=\pi / 2+k \pi, k \in \mathbb{Z}$, we have $a(\alpha)=0, a(\alpha), b(\alpha)= \pm 1 / 2, c(\alpha)=1$, and our transform turns out to be the Fourier one. Throughout this paper, for shortness, the constants $a(\alpha), b(\alpha)$ and $c(\alpha)$ will be simply denoted by $a, b$ and $c$.

Let us recall the definition of normalized Hermite functions $\phi_{n}$, for $n \in \mathbb{N}$, which are defined as

$$
\phi_{n}(x):=(-1)^{n}\left(2^{n} n ! \sqrt{\pi}\right)^{-\frac{1}{2}} e^{\frac{x^{2}}{2}} \frac{d^{n}}{d x^{n}} e^{-x^{2}} .
$$

In the sequel, for $f \in L^{1}(\mathbb{R})$, we define the norm $\|f\|_{1}$ as $\|f\|_{1}:=\frac{1}{\sqrt{2 \pi|\sin \alpha|}} \int_{\mathbb{R}}|f(x)| d x$.

Theorem 1 If $f, g \in L^{1}(\mathbb{R})$, then the transform

$$
(f \odot g)(x) \quad:=\frac{-a b c}{\pi} \int_{\mathbb{R}^{2}} e^{i a\left(u^{2}+v^{2}-x^{2}\right)-2 a^{2} b^{2}(x-u-v)^{2}} f(u) g(v) d u d v
$$

defines a convolution which admits the norm inequality $\|f \odot g\|_{1} \leq\|f\|_{1}\|g\|_{1}$ and the following factorization identity:

$$
\mathcal{F}_{\alpha}[f \odot g](x)=\psi(x) \mathcal{F}_{\alpha}[f](x) \mathcal{F}_{\alpha}[g](x),
$$

where $\psi(x):=e^{-\frac{1}{2} x^{2}-i a x^{2}}$. In other words, the product $f \odot g$ defines a new function belonging to $L^{1}(\mathbb{R})$, and satisfies the convolution theorem for the FrFT up to the weight function (factor) $\psi$.

Proof. We start by proving the norm inequality. Note that $|c|=|\sin \alpha|^{-\frac{1}{2}}$. Performing the change of variables $a b(x-u-v)=t$, we have

$$
\begin{aligned}
\int_{\mathbb{R}}|(f \odot g)(x)| d x & \leq \frac{a b}{\pi \sqrt{|\sin \alpha|}} \int_{\mathbb{R}^{3}}|f(u)||g(v)| e^{-2 a^{2} b^{2}(x-u-v)^{2}} d u d v d x=\frac{1}{\pi \sqrt{|\sin \alpha|}} \int_{\mathbb{R}^{2}}|f(u)| d u|g(v)| d v \int_{\mathbb{R}} e^{-2 t^{2}} d t \\
& =\frac{1}{\sqrt{2 \pi|\sin \alpha|}} \int_{\mathbb{R}}|f(u)| d u \int_{\mathbb{R}}|g(v)| d v=\sqrt{2 \pi|\sin \alpha|}\|f\|_{1}\|g\|_{1},
\end{aligned}
$$

which proves the norm inequality. Obviously, this inequality ensures immediately that the new function defined by (3) belongs to $L^{1}(\mathbb{R})$.

Now we will prove the factorization property (4). Here and in what follows, we will use the following known identity $\frac{1}{\sqrt{2 \pi}} \int_{\mathbb{R}} e^{i x t} e^{-k t^{2}} d t=\frac{1}{\sqrt{2 k}} e^{-\frac{1}{4 k} x^{2}}(k>0)$ for every $x \in \mathbb{R}$ (see [29, 32]). By using the last identity, with $k=1 / 2$, we have

$$
\begin{aligned}
\psi(x) \mathcal{F}_{\alpha}[f](x) \mathcal{F}_{\alpha}[g](x) & =e^{-\frac{1}{2} x^{2}-i a x^{2}} \frac{c}{\sqrt{2 \pi}} \int_{\mathbb{R}} e^{i a\left(x^{2}+u^{2}-2 x u b\right)} f(u) d u \frac{c}{\sqrt{2 \pi}} \int_{\mathbb{R}} e^{i a\left(x^{2}+v^{2}-2 x v b\right)} g(v) d v \\
& =e^{-i a x^{2}} \frac{1}{\sqrt{2 \pi}} \int_{\mathbb{R}} e^{-\frac{1}{2} t^{2}+i x t} d t \frac{c}{\sqrt{2 \pi}} \int_{\mathbb{R}} e^{i a\left(x^{2}+u^{2}-2 x u b\right)} f(u) d u \frac{c}{\sqrt{2 \pi}} \int_{\mathbb{R}} e^{i a\left(x^{2}+v^{2}-2 x v b\right)} g(v) d v \\
& =\frac{c^{2}}{2 \pi \sqrt{2 \pi}} e^{-i a x^{2}} \int_{\mathbb{R}^{3}} e^{i a\left[2 x^{2}+u^{2}+v^{2}-2 x b(u+v)\right]} e^{-\frac{1}{2} t^{2}+i x t} f(u) g(v) d u d v d t \\
& =\frac{c^{2}}{2 \pi \sqrt{2 \pi}} \int_{\mathbb{R}^{3}} e^{i a\left[x^{2}+u^{2}+v^{2}-2 x b\left(u+v-\frac{t}{2 a b}\right)\right]} e^{-\frac{1}{2} t^{2}} f(u) g(v) d u d v d t
\end{aligned}
$$


Changing variables by $u=u, v=v, s=u+v-\frac{t}{2 a b}$, we have

$$
\begin{aligned}
\psi(x) \mathcal{F}_{\alpha}[f](x) \mathcal{F}_{\alpha}[g](x) & =\frac{-a b c^{2}}{\pi \sqrt{2 \pi}} \int_{\mathbb{R}^{3}} e^{i a\left[x^{2}+u^{2}+v^{2}-2 x b s\right]} e^{-2 a^{2} b^{2}(s-u-v)^{2}} f(u) g(v) d u d v d s \\
& =\frac{c}{\sqrt{2 \pi}} \int_{\mathbb{R}} e^{i a\left(x^{2}+s^{2}-2 x b s\right)}\left\{\frac{-a b c}{\pi} \int_{\mathbb{R}^{2}} e^{i a\left(u^{2}+v^{2}-s^{2}\right)-2 a^{2} b^{2}(s-u-v)^{2}} f(u) g(v) d u d v\right\} d s \\
& =\mathcal{F}_{\alpha}\left\{\frac{-a b c}{\pi} \int_{\mathbb{R}^{2}} e^{i a\left(u^{2}+v^{2}-s^{2}\right)-2 a^{2} b^{2}(s-u-v)^{2}} f(u) g(v) d u d v\right\}(x)=\mathcal{F}_{\alpha}[f \odot g](x) .
\end{aligned}
$$

Theorem 2 If $f, g \in L^{1}(\mathbb{R})$, then the following transform defines a convolution followed by the norm inequality and factorization identity:

$$
\begin{gathered}
(f \otimes g)(x):=\frac{e^{i n \alpha} c^{2}}{2 \pi} \int_{\mathbb{R}^{2}} e^{2 i a\left(u^{2}+v^{2}-x u-x v+u v\right)} \phi_{n}(x-u-v) f(u) g(v) d u d v, \\
\|f \otimes g\|_{1} \leq\left\|\phi_{n}\right\|_{1} \quad\|f\|_{1}\|g\|_{1}, \quad \mathcal{F}_{\alpha}[f \otimes g](x)=\Phi_{n}(x) \mathcal{F}_{\alpha}[f](x) \mathcal{F}_{\alpha}[g](x),
\end{gathered}
$$

where $\Phi_{n}(x):=e^{-i 2 a x^{2}} \phi_{n}(x)(c f .(2))$. Thus, the new product $f \otimes g$ defines a function belonging to $L^{1}(\mathbb{R})$, and satisfies the convolution theorem for the FrFT associated with the Hermite function $\phi_{n}$ scaled by the chirp $e^{-i 2 a x^{2}}$.

Proof. We shall prove the inequality in (6). Changing variables $u=u, v=v$, and $t=x-u-v$, and using $\|\cdot\|_{1}$, we have

$$
\begin{aligned}
\int_{\mathbb{R}}|(f \otimes g)(x)| d x & \leq \frac{1}{2 \pi|\sin \alpha|} \int_{\mathbb{R}^{3}}|f(u)||g(v)|\left|\phi_{n}(x-u-v)\right| d u d v d x \\
& =\frac{1}{2 \pi|\sin \alpha|} \int_{\mathbb{R}}|f(u)| d u \int_{\mathbb{R}}|g(v)| d v \int_{\mathbb{R}}\left|\phi_{n}(t)\right| d t=\sqrt{2 \pi|\sin \alpha|}\left\|\phi_{n}\right\|_{1}\|f\|_{1}\|g\|_{1},
\end{aligned}
$$

as desired. This inequality implies that the function defined by (5) belongs to $L^{1}(\mathbb{R})$. Now we will prove the factorization property (6). From the definition of the FrFT, we have

$$
\begin{aligned}
\Phi_{n}(x) \mathcal{F}_{\alpha}[f](x) \mathcal{F}_{\alpha}[g](x) & =\frac{e^{-i 2 a x^{2}} \phi_{n}(x)}{\sqrt{2 \pi}} \int_{\mathbb{R}} e^{i a\left(x^{2}+u^{2}-2 x u b\right)} f(u) d u \frac{c^{2}}{\sqrt{2 \pi}} \int_{\mathbb{R}} e^{i a\left(x^{2}+v^{2}-2 x v b\right)} g(v) d v \\
& =\frac{c^{3} e^{-i 2 a x^{2}}}{(2 \pi)^{3 / 2}} \int_{\mathbb{R}} e^{i n \alpha} e^{i a\left(x^{2}+t^{2}-2 x t b\right)} \phi_{n}(t) d t \int_{\mathbb{R}} e^{i a\left(x^{2}+u^{2}-2 x u b\right)} f(u) d u \int_{\mathbb{R}} e^{i a\left(x^{2}+v^{2}-2 x v b\right)} g(v) d v \\
& =\frac{e^{i n \alpha} c^{3}}{(2 \pi)^{\frac{3}{2}}} \int_{\mathbb{R}^{3}} e^{-i 2 a x^{2}} e^{i a\left[3 x^{2}+u^{2}+v^{2}+t^{2}-2 x b(u+v+t)\right]} f(u) g(v) \phi_{n}(t) d u d v d t \\
& =\frac{e^{i n \alpha} c^{3}}{(2 \pi)^{\frac{3}{2}}} \int_{\mathbb{R}^{3}} e^{i a\left[x^{2}+u^{2}+v^{2}+t^{2}-2 x b(u+v+t)\right]} f(u) g(v) \phi_{n}(t) d u d v d t .
\end{aligned}
$$

Performing the change of variables $u=u, v=v$ and $s=u+v+t$, we obtain

$$
\begin{aligned}
\Phi_{n}(x) \mathcal{F}_{\alpha}[f](x) \mathcal{F}_{\alpha}[g](x) & =\frac{e^{i n \alpha} c^{3}}{(2 \pi)^{\frac{3}{2}}} \int_{\mathbb{R}^{3}} e^{i a\left[x^{2}+u^{2}+v^{2}+(s-u-v)^{2}-2 x b s\right]} f(u) g(v) \phi_{n}(s-u-v) d u d v d s \\
& =\frac{c}{\sqrt{2 \pi}} \int_{\mathbb{R}} e^{i a\left(x^{2}+s^{2}-2 x b s\right)}\left\{\frac{e^{i n \alpha} c^{2}}{2 \pi} \int_{\mathbb{R}} e^{2 i a\left(u^{2}+v^{2}-s u-s v+u v\right)} f(u) g(v) \phi_{n}(s-u-v) d u d v\right\} d s \\
& =\mathcal{F}_{\alpha}\left\{\frac{e^{i n \alpha} c^{2}}{2 \pi} \int_{\mathbb{R}^{2}} e^{2 i a\left(u^{2}+v^{2}-s u-s v+u v\right)} f(u) g(v) \phi_{n}(s-u-v) d u d v\right\}(x)=\mathcal{F}_{\alpha}[f \otimes g](x) .
\end{aligned}
$$

Theorem 3 If $f, g \in L^{1}(\mathbb{R})$, then the transform

$$
(f \odot g)(x):=\frac{e^{i n \alpha}}{2 \pi|\sin \alpha|} \int_{\mathbb{R}^{2}} e^{i a\left(2 u^{2}-2 u x-2 u v+2 x v\right)} \phi_{n}(x-u+v) f(u) g(v) d u d v
$$


defines a convolution with the norm inequality $\|f \odot g\|_{1} \leq\left\|\phi_{n}\right\|_{1}\|f\|_{1}\|g\|_{1}$, and the factorization identity

$$
\mathcal{F}_{\alpha}[f \odot g](x)=\phi_{n}(x) \mathcal{F}_{\alpha}[f](x) \mathcal{F}_{-\alpha}[g](x) .
$$

This means that the product $f \circ g$ defines a function belonging to $L^{1}(\mathbb{R})$, and satisfies the convolution theorem associated with the FrFT and its inverse (IFrFT) with the factor $\phi_{n}(x)$.

Proof. The norm inequality can be proved in the same way as in the proof of the inequality in (6), and so we omit the proof. We will prove the factorization property (8). By the definition of FrFT, we have

$$
\begin{aligned}
\phi_{n}(x) \mathcal{F}_{\alpha}[f](x) & \mathcal{F}_{-\alpha}[g](x)=e^{i n \alpha} \frac{\sqrt{1-i \cot \alpha}}{\sqrt{2 \pi}} \frac{\sqrt{1+\cot ^{2} \alpha}}{2 \pi} \int_{\mathbb{R}^{3}} e^{i a\left[x^{2}+t^{2}+u^{2}+x^{2}-x^{2}-v^{2}-2 x b(t+u-v)\right]} f(u) g(v) \phi_{n}(t) d u d v d t \\
& =e^{i n \alpha} \frac{\sqrt{1-i \cot \alpha}}{\sqrt{2 \pi}} \frac{\sqrt{1+\cot ^{2} \alpha}}{2 \pi} \int_{\mathbb{R}^{3}} e^{i a\left[x^{2}+t^{2}+u^{2}-v^{2}-2 x b(t+u-v)\right]} f(u) g(v) \phi_{n}(t) d u d v d t \\
& =e^{i n \alpha} \frac{\sqrt{1-i \cot \alpha}}{\sqrt{2 \pi}} \frac{\sqrt{1+\cot ^{2} \alpha}}{2 \pi} \int_{\mathbb{R}^{3}} e^{i a\left[x^{2}+(s-u+v)^{2}+u^{2}-v^{2}-2 x b s\right]} f(u) g(v) \phi_{n}(s-u+v) d u d v d s \\
& =e^{i n \alpha} \frac{\sqrt{1-i \cot \alpha}}{\sqrt{2 \pi}} \frac{1}{2 \pi|\sin \alpha|} \int_{\mathbb{R}} e^{i a\left(x^{2}+s^{2}-2 x b s\right)}\left(\int_{\mathbb{R}^{2}} e^{i a\left(2 u^{2}-2 u s-2 u v+2 s v\right)} f(u) g(v) \phi_{n}(s-u+v) d u d v\right) d s \\
& =\mathcal{F}_{\alpha}[f \odot g](x),
\end{aligned}
$$

which proves the theorem.

We notice that convolutions (3) and (5) satisfy the commutative, associative and distributive properties. However, ๑, defined by (7), does not satisfy any of those properties (just because it is a convolution associated with the two transforms $\mathcal{F}_{\alpha}$ and $\mathcal{F}_{-\alpha}$ ).

\section{YOUNG'S CONVOLUTION INEQUALITIES}

In [31], Singh and Saxena gave a meaningful and detailed comparison between their convolutions and the convolutions previously constructed. Here, we shall start by discussing the possible framework for the convolutions in $[3,4,14,31$, $33,35]$ and then will make a consequent comparative analysis.

We observe that in [3], Almeida obtained some convolutions associated with FrFT, under some assumptions on the domain space (as proved by [4]). In [35], Zayed introduced two interesting convolutions, associated with FrFT, denoted by $\star$ and $\otimes$. The process of $f \star g$ can be interpreted as a signal $f$, scaled by the factor $e^{i a x^{2}}$, which convolutes in the Fourier sense with the signal $g$, scaled by the same factor $e^{i a x^{2}}$, up to the weight function $c e^{-i x^{2}} / \sqrt{2 \pi}$. The convolution $\otimes$ was built by using the same idea but for a scaled factor $e^{-i a x^{2}}$ and a weighted factor $c e^{i x^{2}} / \sqrt{2 \pi}$.

It took eight years so that the convolution theorems for the linear canonical transforms (LCT) were provided by Deng et al. in [14]. Having in mind the Zayed's idea for FrFT, the convolutions in [14] can be seen as new versions for LCT of what was known for the FrFT case. Despite the cumbrousness of expressions, they may be applicable in some practical models.

In 2009, Wei et al., in [33], offered the concept on $\tau$-generalized translation of a signal, and presented a convolution expression associated with the LCT. We see that the convolution expression in [33] was constructed by the time shift/modulation properties of the LCT. This is why this convolution is elegant and flexible, and the transform (LCT) of the convolution has no scaling functions. At this point we should clarify that the LCT has an advantage over other ones due to its flexible use. However, its computation may be more expensive since it contains four parameters.

In [31], the authors developed a convolution theorem and a product theorem for the FrFT. In this work we may also find a somehow detailed comparison between different convolutions.

The authors of [4] gave two new convolutions associated with FrFT in which there are a time-delay and a shift. As a result, the transform (FrFT) of each one of convolutions is a simple product of two image functions scaled by the weight functions $e^{i\left( \pm x-a x^{2}\right)}$.

Taking into consideration those works, we realizer that the structure of those convolutions are still in the framework of the known Fourier convolution when scaled by some chirps. In this work, we shall be out of that framework 
when looking for new convolutions. As a result, infinitely many convolutions related to Hermite functions have been found (as already presented in the previous section), and some natural consequences will be now deduced in this section and also in the next section.

For short, put $\mathcal{E}_{\mathrm{ch}}(t):=e^{i a t^{2}}, \mathcal{E}_{\mathrm{gd}}(t):=e^{-2 a^{2} b^{2} t^{2}}$, where $\mathcal{E}_{\mathrm{ch}}, \mathcal{E}_{\mathrm{gd}}$ are the chirp and Gaussian functions, respectively. Then, $\mathcal{E}(s, u, v):=e^{i a\left(u^{2}+v^{2}-s^{2}\right)-2 a^{2} b^{2}(s-u-v)^{2}}=\mathcal{E}_{\mathrm{ch}}(u) \mathcal{E}_{\mathrm{ch}}(v)\left[\mathcal{E}_{\mathrm{ch}}(s)\right]^{-1} \mathcal{E}_{\mathrm{gd}}(s-u-v)$. Thus, we can say that $\mathcal{E}$ is a product of the three chirps $\mathcal{E}_{\mathrm{ch}}(u), \mathcal{E}_{\mathrm{ch}}(v),\left[\mathcal{E}_{\mathrm{ch}}(s)\right]^{-1}$ and the Gaussian distribution $\mathcal{E}_{\mathrm{gd}}$. As presented, there is a great diversity of constructed convolutions. Most of them are flexible as there is the presence of delays and shifts in the Hermite function, and chirp functions are in full forms. Roughly speaking, the proposed convolutions are combined by three chirps with independent coordinates and a Gaussian or Hermite distribution of signals. This supplement can meet different mathematical models for engineering problems. For serving those purposes, we study some classes of general integral equations generated by Gaussian and Hermite kernels, and obtain the explicit form of solutions which will be presented in the next section.

We will now prove two norm inequalities for the convolutions here introduced. For this purpose, we first recall the Minkowski's integral inequality

$$
\left[\int_{\Omega_{2}}\left|\int_{\Omega_{1}} F(x, y) d \mu_{1}(x)\right|^{r} d \mu_{2}(y)\right]^{\frac{1}{r}} \leq \int_{\Omega_{1}}\left(\int_{\Omega_{2}}|F(x, y)|^{r} d \mu_{2}(y)\right)^{\frac{1}{r}} d \mu_{1}(x),
$$

where the function $F(\cdot, \cdot): \Omega_{1} \times \Omega_{2} \longrightarrow \mathbb{C}$ is measurable on two measure spaces $\left(\Omega_{1}, \mu_{1}\right)$ and $\left(\Omega_{2}, \mu_{2}\right)$. In what follows, let $1 \leq p, q, r \leq \infty$ such that $\frac{1}{p}+\frac{1}{q}=\frac{1}{r}+1$. Naturally, the Banach spaces here involved are $L^{p}(\mathbb{R}), L^{q}(\mathbb{R})$ and $L^{r}(\mathbb{R})$.

Theorem 4 Let $p, q, r \in[1, \infty]$ satisfy $\frac{1}{p}+\frac{1}{q}=\frac{1}{r}+1$. Denoting by $\circledast$ any of the three previously considered convolutions $\odot, \otimes$ and $\odot$, it holds

$$
\begin{aligned}
& \|f \circledast g\|_{r} \leq C_{1}\|f\|_{p}\|g\|_{q}, \text { provided } f \in L^{p}(\mathbb{R}), g \in L^{q}(\mathbb{R}), \\
& \|f \circledast g\|_{s} \leq C_{2}\|f\|_{1}\|g\|_{1}, \text { for any } s \geq 1, \text { provided } f, g \in L^{1}(\mathbb{R}),
\end{aligned}
$$

where $C_{1}$ and $C_{2}$ are some positive constants.

Proof. We shall deduce the inequalities (10)-(11) for the convolution (3), and will omit the cases of (5) and (7), since the proofs are analogous.

The key point on the proofs comes from the Gaussian and Hermite functions $\mathcal{E}_{\mathrm{gd}}$ and $\psi_{n}$, which are rapidly decreasing functions. Let us start by the proof of (10). Performing the change of variable $t:=u+v$, we have

$$
\begin{aligned}
h(s) & :=\frac{-a b c}{\pi} \iint_{\mathbb{R}^{2}} \mathcal{E}_{\mathrm{ch}}(u) \cdot \mathcal{E}_{\mathrm{ch}}(v) \cdot\left[\mathcal{E}_{\mathrm{ch}}(s)\right]^{-1} \cdot \mathcal{E}_{\mathrm{gd}}(s-u-v) f(u) g(v) d u d v \\
& =\frac{-a b c}{\pi} \int_{\mathbb{R}}\left[\mathcal{E}_{\mathrm{ch}}(s)\right]^{-1} \cdot \mathcal{E}_{\mathrm{gd}}(s-t) d t\left(\int_{\mathbb{R}}\left[\mathcal{E}_{\mathrm{ch}}(t-v) f(t-v)\right] \cdot\left[\mathcal{E}_{\mathrm{ch}}(v) g(v)\right] d v\right)=\frac{-a b c}{\pi \mathcal{E}_{\mathrm{ch}}(s)} \int_{\mathbb{R}} \mathcal{E}_{\mathrm{gd}}(s-t) F(t) d t,(12
\end{aligned}
$$

where $F(t):=\int_{\mathbb{R}}\left[\mathcal{E}_{\mathrm{ch}}(t-v) f(t-v)\right] .\left[\mathcal{E}_{\mathrm{ch}}(v) g(v)\right] d v$. Evidently, $\mathcal{E}_{\mathrm{ch}} f \in L^{p}(\mathbb{R}), \mathcal{E}_{\mathrm{ch}} g \in L^{q}(\mathbb{R})$. Applying the wellknown Young's convolution inequality for the Fourier case [6,7], we have $F \in L^{r}(\mathbb{R})$. Remind that $\left|\mathcal{E}_{\mathrm{ch}}\right|=1$, and $\mathcal{E}_{\mathrm{gd}} \in L^{1}(\mathbb{R})$. Again, applying the Young's inequality for $\mathcal{E}_{\mathrm{gd}}$ and $F$, within the case $\frac{1}{r}+\frac{1}{1}=\frac{1}{r}+1$, we derive that the function defined by the right-hand side of (12) belongs to $L^{r}(\mathbb{R})$. This means that $h \in L^{r}(\mathbb{R})$.

Let us now turn to the proof of (11). Note that $\mathcal{E}_{\mathrm{gd}} \in L^{s}(\mathbb{R})$, for any $s>0$, and $\int_{\mathbb{R}}\left|\mathcal{E}_{\mathrm{gd}}( \pm x \pm u \pm v)\right|^{s} d x=\left\|\mathcal{E}_{\mathrm{gd}}\right\|_{s}^{s}$ $(u, v$ are fixed in $\mathbb{R})$. Applying (9), it follows

$$
\begin{aligned}
& {\left[\left.\int_{\mathbb{R}}\left|\int_{\mathbb{R}^{2}} \mathcal{E}_{\mathrm{gd}}(x-u-v) f(u) g(v) d u d v\right|^{s} d x\right|^{1 / s} \leq \int_{\mathbb{R}^{2}}\left(\int_{\mathbb{R}}\left|\mathcal{E}_{\mathrm{gd}}(x-u-v)\right|^{s} \cdot|f(u)|^{s} \cdot|g(v)|^{s} d x\right)^{1 / s} d u d v\right.} \\
& =\int_{\mathbb{R}^{2}}\left(\int_{\mathbb{R}}\left|\mathcal{E}_{\mathrm{gd}}(x-u-v)\right|^{s} d x\right)^{1 / s} \cdot|f(u)| \cdot|g(v)| d u d v=\left\|\mathcal{E}_{\mathrm{gd}}\right\|_{s} \cdot \int_{\mathbb{R} 2}|f(u)| \cdot|g(v)| d u d v=\left\|\mathcal{E}_{\mathrm{gd}}\right\|_{s} \cdot\|f\|_{1} \cdot\|g\|_{1},
\end{aligned}
$$

and so (11) is obtained. 
We would like to point out that the last result exposes a notable difference between the image and domain spaces of the proposed convolutions and the ones of previously constructed convolutions, associated with FrFT and LCT. Namely, here the convolutions (3), (5) and (7) possess their own direct Young's convolution inequalities (10), as well as (11). In other words, if $f \in L^{p}(\mathbb{R})$ and $g \in L^{q}(\mathbb{R})$, then each one of the proposed convolutions defines a function in $L^{r}(\mathbb{R})$, where $1 / p+1 / q=1 / r+1$. Moreover, if $f, g \in L^{1}(\mathbb{R})$, then each one of those convolutions also defines a function in $L^{s}(\mathbb{R})$, for any $s \geq 1$. In notation, we may write this as $L^{p}(\mathbb{R}) \circledast L^{q}(\mathbb{R}) \subseteq L^{r}(\mathbb{R}), L^{1}(\mathbb{R}) \circledast L^{1}(\mathbb{R}) \subseteq L^{s}(\mathbb{R})$, for any $s \geq 1$.

Remark 5 (a) Letting $p=q=r=1$ in (10), or $s=1$ in (11), we retrieve the norm inequalities proved by Theorems 1, 2, and 3.

(b) Choosing $s=2$ in (11), we see that: if $f, g \in L^{1}(\mathbb{R})$, then the convolution defines a function in the space $L^{1}(\mathbb{R}) \cap L^{2}(\mathbb{R})$. This result is suitable with the known fact that a convolution $f * g$, roughly speaking, inherits the best properties of both $f$ and $g$. In particular, since a convolution can be seen as a process of filtering, averaging, inner product or smoothing, in a certain sense, the Young type convolution inequality (11) is a distinctive feature of the proposed convolutions. This may be of interest since (11) exhibits a remarkable difference in comparison with the known ones which are associated with the Fourier case. Moreover, when focusing on the case $L^{2}(\mathbb{R})$, and having in mind that the Hermite functions constitute a standard-orthogonal basis system in the Hilbert space $L^{2}(\mathbb{R})$, we notice that those convolutions have a great potential of application in Harmonic Analysis.

(c) The appearance of Hermite functions in the above convolution kernels plays a key role in the potential of application of those convolutions. In particular, the Young's inequality (11) is already a typical characteristic of such a structure.

Theorem 6 The Banach space $L^{1}(\mathbb{R})$, equipped with the convolution multiplication (3) or (5), becomes a commutative normed algebra.

Proof. After having the above results, we can complete this proof by using a very direct way. Indeed, by Theorems 1,2 , the convolution multiplications (3) and (5) are closed in $L^{1}(\mathbb{R})$, and have the commutative and associative properties.

We recall that this occurs also for generalized convolutions of Fourier transform; cf. [18, 19].

\section{CLASSES OF CONVOLUTION INTEGRAL EQUATIONS}

In this section, we establish necessary and sufficient conditions for the solvability of some classes of convolution equations associated with the FrFT. Moreover, under appropriate conditions, we obtain the corresponding explicit solutions of those integral equations.

We start by considering the following type of integral equation, in the Banach space $L^{1}(\mathbb{R})$ :

$$
\lambda \varphi(s)+(k \odot \varphi)(s)=f(s)
$$

where $\lambda \in \mathbb{C}$ and $k \in L^{1}(\mathbb{R})$ are given, and $\varphi$ is to be determined (in this space). We shall use the notation $A(s):=$ $\lambda+\psi(s) \mathcal{F}_{\alpha}[k](s)$, where $\psi$ is the function introduced in Theorem 1. The following proposition is useful for proving the characterization of the solvability of equation (13), which will be given in Theorem 8 .

Proposition $7 \quad$ (1) If $\lambda \neq 0$, then $A(s) \neq 0$ for every $s$ outside a finite interval.

(2) If $A(s) \neq 0$ for every $s \in \mathbb{R}$, then the function $1 / A(s)$ is bounded and continuous on $\mathbb{R}$.

Proof. (1) By the Riemann-Lebesgue lemma, the function $A(s)$ is continuous on $\mathbb{R}$ and

$$
\lim _{|s| \rightarrow \infty} A(s)=\lambda \neq 0,
$$

i.e., $A(s)$ takes the value $\lambda$ at infinity. Since $\lambda \neq 0$ and $A(s)$ is continuous, there exists an $R>0$ such that $A(s) \neq 0$ for every $|s|>R$. Proposition (1) is proved. 
(2) Due to the continuity of $A$ and (14), there exist $R_{0}>0, \epsilon_{1}>0$ such that $\inf _{|s|>R_{0}}|A(s)|>\epsilon_{1}$. As $A$ is continuous and not vanishing on the compact set $S\left(0, R_{0}\right)=\left\{s \in \mathbb{R}:|s| \leq R_{0}\right\}$, there exists $\epsilon_{2}>0$ such that $\inf _{|s| \leq R_{0}}|A(s)|>\epsilon_{2}$. We deduce $\sup _{s \in \mathbb{R}} \frac{1}{|A(s)|} \leq \max \left\{\frac{1}{\epsilon_{1}}, \frac{1}{\epsilon_{2}}\right\}<\infty$. This implies that the function $1 /|A(s)|$ is continuous and bounded on $\mathbb{R}$. The proposition is proved.

Theorem 8 Assume that $A(s) \neq 0$ for every $s \in \mathbb{R}$, and one of the following conditions is satisfied:

(i) $\lambda \neq 0$, and $\mathcal{F}_{\alpha}[f] \in L^{1}(\mathbb{R})$;

(ii) $\lambda=0$, and $\frac{\mathcal{F}_{\alpha}[f]}{\mathcal{F}_{\alpha}[k]} \in L^{1}(\mathbb{R})$.

Then, equation (13) has a solution in $L^{1}(\mathbb{R})$ if and only if $\mathcal{F}_{-\alpha}\left[\mathcal{F}_{\alpha}[f] / A\right] \in L^{1}(\mathbb{R})$. If this is the case, then the solution is given by $\varphi:=\mathcal{F}_{-\alpha}\left[\mathcal{F}_{\alpha}[f] / A\right]$.

Proof. Let us first assume that (i) is fulfilled.

Necessity. Suppose that equation (13) has a solution $\varphi \in L^{1}(\mathbb{R})$. Applying $\mathcal{F}_{\alpha}$ to both sides of equation (13) and using the factorization identity in Theorem 1, we obtain $A(s) \mathcal{F}_{\alpha}[\varphi](s)=\mathcal{F}_{\alpha}[f](s)$. Since $A(s) \neq 0$ for every $s \in \mathbb{R}$, $\mathcal{F}_{\alpha}[\varphi]=\frac{\mathcal{F}_{\alpha}[f]}{A}$. As the function $1 / A(s)$ is bounded and continuous on $\mathbb{R}$ (cf. Proposition 7 ) and $\mathcal{F}_{\alpha}[f] \in L^{1}(\mathbb{R})$, we deduce that $\left(\mathcal{F}_{\alpha} f / A\right) \in L^{1}(\mathbb{R})$. We can now apply the inverse transform of $\mathcal{F}_{\alpha}$ to $\mathcal{F}_{\alpha}[\varphi]=\frac{\mathcal{F}_{\alpha}[f]}{A}$ for obtaining the solution as stated in the theorem. The necessity part is proved.

Sufficiency. Consider the function $\varphi:=\mathcal{F}_{-\alpha}\left[\frac{\mathcal{F}_{\alpha}[f]}{A}\right]$. By the assumption, $\varphi \in L^{1}(\mathbb{R})$. Hence, $\mathcal{F}_{\alpha}[\varphi]=\mathcal{F}_{\alpha}[f] / A$. Equivalently, $A\left(\mathcal{F}_{\alpha}[\varphi]\right)=\mathcal{F}_{\alpha}[f]$. Thanks to the factorization identity, we have $\mathcal{F}_{\alpha}[\lambda \varphi+(k \odot \varphi)]=\mathcal{F}_{\alpha}[f]$. By the uniqueness theorem of $\mathcal{F}_{\alpha}$, we conclude that $\varphi$ fulfills equation (13) for almost every $s \in \mathbb{R}$. Item (i) is proved.

Since $|\psi(x)|=1$, the function $1 / \psi$ is continuous and bounded on $\mathbb{R}$. Hence, $\mathcal{F}_{\alpha}[f] / \mathcal{F}_{\alpha}[k] \in L^{1}(\mathbb{R})$ if and only if $\mathcal{F}_{\alpha}[f] /\left(\psi \cdot \mathcal{F}_{\alpha}[k]\right) \in L^{1}(\mathbb{R})$. Therefore, item (ii) can be proved similarly to that of item (i). The theorem is proved.

By using the same method as we just did in Theorem 8, we were able to produce a corresponding characterization of the solvability of another class of integral equations, and provide their solutions. We shall formulate the result, and omit the proof as it can be deduced analogously to the last one.

\section{Theorem 9 Consider the equation}

$$
\lambda \varphi(s)+(k \otimes \varphi)(s)=f(s),
$$

and assume that $B_{n}(s):=\lambda+\psi_{n}(s) \mathcal{F}_{\alpha}[k](s) \neq 0$ for every $s \in \mathbb{R}$, and that one of the following conditions is satisfied:

$$
\text { (i) } \lambda \neq 0, \quad \text { and } \quad \mathcal{F}_{\alpha}[f] \in L^{1}(\mathbb{R}) ; \quad \text { (ii) } \lambda=0, \quad \text { and } \quad \frac{\mathcal{F}_{\alpha}[f]}{\mathcal{F}_{\alpha}[k]} \in L^{1}(\mathbb{R})
$$

Equation (15) has solution in $L^{1}(\mathbb{R})$ if and only if $\mathcal{F}_{-\alpha}\left[\mathcal{F}_{\alpha}[f] / B_{n}\right] \in L^{1}(\mathbb{R})$. Moreover, under the conditions of solvability, the solution is given by $\varphi=\mathcal{F}_{-\alpha}\left[\mathcal{F}_{\alpha} f / B\right]$.

We will now consider integral equations whose kernel is generated by two Hermite functions. In particular, let $\phi_{m}, \phi_{n}$ be given Hermite functions, and let $k_{1}, k_{2}$ be functions belonging to $L^{1}(\mathbb{R})$. For shortness of the formulas, we write $\mathcal{E}_{\mathrm{m}-\mathrm{ch}}(u, v, s):=e^{i m \alpha} \cdot e^{i 2 a\left(v^{2}+u s-u v-s v\right)}$ for $m \in \mathbb{N}$. We shall present a necessary and sufficient condition for solving a class of integral equations of the form

$$
\lambda \varphi(s)+\frac{1}{2 \pi|\sin \alpha|} \int_{\mathbb{R}^{2}}\left[\mathcal{E}_{\mathrm{m}-\mathrm{ch}}(u, v, s) \phi_{m}(s+u-v) k_{1}(u)+\mathcal{E}_{\mathrm{n}-\mathrm{ch}}(v, u, s) \cdot \phi_{n}(s-u+v) k_{2}(u)\right] \varphi(v) d u d v=p(s),
$$

where $\lambda \in \mathbb{C}$ and $p \in L^{1}(\mathbb{R})$ are given, and $\varphi(s)$ is the unknown function in $L^{1}(\mathbb{R})$.

Note that $\mathcal{E}_{\mathrm{m}-\mathrm{ch}}$ is a chip and $\left|\mathcal{E}_{\mathrm{m}-\mathrm{ch}}\right|=1$. We derive $k_{1} \cdot\left[\mathcal{E}_{\mathrm{m}-\mathrm{ch}}\right]^{-1} \in L^{1}(\mathbb{R})$ and (or) $k_{2} \cdot\left[\mathcal{E}_{\mathrm{n}-\mathrm{ch}}\right]^{-1} \in L^{1}(\mathbb{R})$ if and only if $k_{1} \in L^{1}(\mathbb{R})$ and (or) $k_{2} \in L^{1}(\mathbb{R})$, respectively. Therefore, the appearance of the factor $\frac{1}{2 \pi|\sin \alpha|}$ and the chirps $\mathcal{E}_{\mathrm{m}-\mathrm{ch}}, \mathcal{E}_{\mathrm{n}-\mathrm{ch}}$ in the kernel of equation (16) is not artificial. In fact, it is just convenient for the direct use of the convolutions, because that factor and the chirps can be merged into the functions $k_{1}, k_{2}$. Indeed, for any $k_{1}, k_{2} \in L^{1}(\mathbb{R})$, we can write $k_{1}=\mathcal{E}_{\mathrm{m}-\mathrm{ch}} \cdot\left[\left(\mathcal{E}_{\mathrm{m}-\mathrm{ch}}\right)^{-1} k_{1}\right]:=\mathcal{E}_{\mathrm{m}-\mathrm{ch}} . \widetilde{k}_{1}, k_{2}=\mathcal{E}_{\mathrm{n}-\mathrm{ch}} \cdot\left[\left(\mathcal{E}_{\mathrm{n}-\mathrm{ch}}\right)^{-1} k_{2}\right]:=\mathcal{E}_{\mathrm{n}-\mathrm{ch}} \cdot \widetilde{k}_{2}$, where $\widetilde{k}_{1}, \widetilde{k}_{2}$ are also in 
$L^{1}(\mathbb{R})$. That is why equation (16) can be seen as a universal Hermite kernel which includes many integral equations. There are some special cases of (16) which appear in engineering problems (for example, see [16, 17] and references therein).

In view to solve this equation we need an additional convolution operation.

Definition 10 We define the product $\oplus$ by

$$
h(s):=(f \oplus g)(s)=\frac{e^{i m \alpha}}{2 \pi|\sin \alpha|} \int_{\mathbb{R}^{2}} e^{i a\left(2 v^{2}+2 u s-2 u v-2 s v\right)} f(u) g(v) \phi_{m}(s+u-v) d u d v,
$$

which satisfies the following properties:

$$
\|f \oplus g\|_{1} \leq\left\|\phi_{n}\right\|_{1}\|f\|_{1}\|g\|_{1}, \quad \mathcal{F}_{\alpha}[f \oplus g](x)=\phi_{m}(x) \mathcal{F}_{-\alpha}[f](x) \mathcal{F}_{\alpha}[g](x) .
$$

It is necessary to say that the operation defined by (17) can be deduced from that one defined by (7). Indeed, switching the role $u$ and $v$ in the integration of (7), we obtain (17) and the inequality in (18). In this sense, the operation (17) is not a new convolution. Instead, it is just a small adaption in that previous one, but it is enough for our purpose.

For the sake of shortness of notation, let us fix:

$$
\begin{aligned}
D(x) & :=\left[\lambda+\phi_{m}(x)\left(\mathcal{F}_{-\alpha} k_{1}\right)(x)\right]\left[\lambda+\phi_{m}(x)\left(\mathcal{F}_{\alpha} k_{1}\right)(x)\right]-\left[\phi_{n}(x)\left(\mathcal{F}_{\alpha} k_{2}\right)(x)\right]\left[\phi_{n}(x)\left(\mathcal{F}_{-\alpha} k_{2}\right)(x)\right] ; \\
D_{\mathcal{F}_{\alpha}}(x) & :=\left(\mathcal{F}_{\alpha} p\right)(x)\left[\lambda+\phi_{m}(x)\left(\mathcal{F}_{\alpha} k_{1}\right)(x)\right]-\phi_{n}(x)\left(\mathcal{F}_{\alpha} k_{2}\right)(x)\left(\mathcal{F}_{-\alpha} p\right)(x) ; \\
D_{\mathcal{F}_{-\alpha}}(x) & :=\left(\mathcal{F}_{-\alpha} p\right)(x)\left[\lambda+\phi_{m}(x)\left(\mathcal{F}_{-\alpha} k_{1}\right)(x)\right]-\phi_{n}(x)\left(\mathcal{F}_{-\alpha} k_{2}\right)(x)\left(\mathcal{F}_{\alpha} p\right)(x) .
\end{aligned}
$$

Proposition 11 is useful for proving Theorem 12. However, let us omit the proof of Proposition 11 as it is analogous to that of Proposition 7.

Proposition 11 (1) If $\lambda \neq 0$, then $D(x) \neq 0$ for every $x$ outside a finite interval.

(2) Assume that $\lambda \neq 0$ and $D(x) \neq 0$ for every $x \in \mathbb{R}$, then the function $1 / D(x)$ is bounded and continuous on $\mathbb{R}$.

Theorem 12 Assume that $D(x) \neq 0$ for every $x \in \mathbb{R}$, and one of the following conditions is satisfied:

(i) $\lambda \neq 0$, and $\mathcal{F}_{\alpha}[p], \mathcal{F}_{-\alpha}[p] \in L^{1}(\mathbb{R})$;

(ii) $\lambda=0$, and $\frac{D_{\mathcal{F}_{\alpha}}}{D}, \frac{D_{\mathcal{F}_{-\alpha}}}{D} \in L^{1}(\mathbb{R})$.

Then equation (16) has a solution in $L^{1}(\mathbb{R})$ if and only if

$$
\mathcal{F}_{-\alpha}\left[\frac{D_{\mathcal{F}_{\alpha}}}{D}\right]=\mathcal{F}_{\alpha}\left[\frac{D_{\mathcal{F}_{-\alpha}}}{D}\right] \in L^{1}(\mathbb{R}) .
$$

If this is the case, then the solution is given by $\varphi=\mathcal{F}_{-\alpha}\left[\frac{D_{\mathcal{F}_{\alpha}}}{D}\right]$.

A way of proving Theorem 12 is in a certain sense analogous to that of Theorem 8, if considering the combination of a system of two equations.

Proof. Let us first assume that (i) is fulfilled.

Necessity. Suppose that equation (16) has a solution $\varphi \in L^{1}(\mathbb{R})$. Applying $\mathcal{F}_{\alpha}$ to both sides of (16), we obtain

$$
\lambda \mathcal{F}_{\alpha}[\varphi](x)+\phi_{m}(x) \mathcal{F}_{-\alpha}\left[k_{1}\right](x) \mathcal{F}_{\alpha}[\varphi](x)+\phi_{n}(x) \mathcal{F}_{\alpha}\left[k_{2}\right](x) \mathcal{F}_{-\alpha}[\varphi](x)=\mathcal{F}_{\alpha}[p](x)
$$

Equivalently, $\left[\lambda+\phi_{m}(x) \mathcal{F}_{-\alpha}\left[k_{1}\right](x)\right] \mathcal{F}_{\alpha}[\varphi](x)+\left[\phi_{n}(x) \mathcal{F}_{\alpha}\left[k_{2}\right](x)\right] \mathcal{F}_{-\alpha}[\varphi](x)=\mathcal{F}_{\alpha}[p](x)$. In this identity, by replacing $\alpha$ by $-\alpha$ we receive a system of two functional equations

$$
\left\{\begin{array}{l}
{\left[\lambda+\phi_{m}(x) \mathcal{F}_{-\alpha}\left[k_{1}\right](x)\right] \mathcal{F}_{\alpha}[\varphi](x)+\left[\phi_{n}(x) \mathcal{F}_{\alpha}\left[k_{2}\right](x)\right] \mathcal{F}_{-\alpha}[\varphi](x)=\mathcal{F}_{\alpha}[p](x)} \\
{\left[\phi_{n}(x) \mathcal{F}_{-\alpha}\left[k_{2}\right](x)\right] \mathcal{F}_{\alpha}[\varphi](x)+\left[\lambda+\phi_{m}(x) \mathcal{F}_{\alpha}\left[k_{1}\right](x)\right] \mathcal{F}_{-\alpha}[\varphi](x)=\mathcal{F}_{-\alpha}[p](x)}
\end{array}\right.
$$

The determinants associated with this system are exactly given by (19), (20), and (21). Hence, $\mathcal{F}_{\alpha}[\varphi]=D_{\mathcal{F}_{\alpha}} / D$, $\mathcal{F}_{-\alpha}[\varphi]=D_{\mathcal{F}_{-\alpha}} / D$. Since $\mathcal{F}_{\alpha}[p], \mathcal{F}_{-\alpha}[p] \in L^{1}(\mathbb{R})$, and the functions $\lambda+\phi_{m}(x) \mathcal{F}_{\alpha}\left[k_{1}\right](x), \lambda+\phi_{m}(x) \mathcal{F}_{-\alpha}\left[k_{1}\right](x)$, 
$\phi_{n}(x) \mathcal{F}_{\alpha}\left[k_{2}\right](x), \phi_{n}(x) \mathcal{F}_{-\alpha}\left[k_{2}\right](x)$ are continuous and bounded on $\mathbb{R}$, we have $D_{\mathcal{F}_{\alpha}}, D_{\mathcal{F}_{-\alpha}} \in L^{1}(\mathbb{R})$. As the function $1 / D(x)$ is bounded and continuous on $\mathbb{R}$ (cf. Proposition 11 ) and $D_{\mathcal{F}_{\alpha}}, D_{\mathcal{F}_{-\alpha}} \in L^{1}(\mathbb{R})$, we deduce that $D_{\mathcal{F}_{\alpha}} / D, D_{\mathcal{F}_{-\alpha}} / D \in$ $L^{1}(\mathbb{R})$. We can now apply the inverse transform to obtain the solution as stated in the theorem. The necessity part is therefore proved.

Sufficiency. Consider the function $\varphi=\mathcal{F}_{-\alpha}\left[D_{\mathcal{F}_{\alpha}} / D\right]=\mathcal{F}_{\alpha}\left[D_{\mathcal{F}_{-\alpha}} / D\right] \in L^{1}(\mathbb{R})$. It implies that $\varphi \in L^{1}(\mathbb{R})$. Hence,

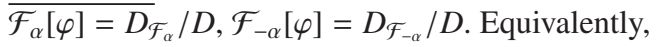

$$
\left\{\begin{array}{l}
\left(\lambda+\phi_{m} \mathcal{F}_{\alpha}\left[k_{1}\right]\right) \mathcal{F}_{\alpha}[p]-\phi_{n} \mathcal{F}_{\alpha}\left[k_{2}\right] \mathcal{F}_{-\alpha}[p]=\mathcal{F}_{\alpha}[\varphi] D \\
\left(\lambda+\phi_{m} \mathcal{F}_{-\alpha}\left[k_{1}\right]\right) \mathcal{F}_{-\alpha}[p]-\phi_{n} \mathcal{F}_{-\alpha}\left[k_{2}\right] \mathcal{F}_{\alpha}[p]=\mathcal{F}_{-\alpha}[\varphi] D
\end{array}\right.
$$

We thus have $\lambda \mathcal{F}_{\alpha}[\varphi](x)+\phi_{m}(x) \mathcal{F}_{-\alpha}\left[k_{1}\right](x) \mathcal{F}_{\alpha}[\varphi](x)+\phi_{n}(x) \mathcal{F}_{\alpha}\left[k_{2}\right](x) \mathcal{F}_{-\alpha}[\varphi](x)=\mathcal{F}_{\alpha}[p]$. Due to the factorization identities for $\oplus$ and $\odot$, we have $\mathcal{F}_{\alpha}\left[\lambda \varphi+\left(k_{1} \oplus \varphi\right)+\left(k_{2} \odot \varphi\right)\right]=\mathcal{F}_{\alpha}[p]$. By the uniqueness theorem of $\mathcal{F}_{\alpha}$ we conclude that $\varphi$ fulfills equation (16) for almost every $x \in \mathbb{R}$. Thus, item (i) is proved. The case (ii) may be proved similarly to that of item (i), and so the proof of Theorem 12 is completed.

Example 13 The following equation can serve as a clear illustration of Theorem 8. Consider the convolution equation

$$
\lambda \varphi(x)+(k \odot \varphi)(x)=f(x),
$$

for any $\lambda \in \mathbb{C}$.

We choose $k(x)=e^{-a|x|}$ with $\mathfrak{R}(a)>0, f(x)=e^{-\frac{1}{2} x^{2}}$. It is easily seen that $k, f \in L^{1}(\mathbb{R})$. Let us denote by $K_{\alpha}(x)$ the FrFT of $k$. Obviously, $|\psi(x)|=1$, and for a fixed $\lambda$ the function $M(x)=\lambda+\psi(x) K_{\alpha}(x)$, is bounded and continuous. Let us consider first the case $\lambda \neq 0$. It holds $K_{\alpha} \in L^{1}(\mathbb{R})$. Additionally, note that the function $\psi(x) K_{\alpha}(x)$ is continuous and bounded, and vanishing at infinity. Therefore, if $\lambda$ is sufficiently large, then $M(x) \neq 0$ for every $x$. For example, the assumption that $|\lambda|>\max _{x \in \mathbb{R}}\left|\psi(x) K_{\alpha}(x)\right|$ is a sufficient condition to guarantee that $M(x)$ is a non-vanishing function. Concerning the second assumption, we have $\mathcal{F}_{\alpha}[f](x)=e^{-\frac{1}{2} x^{2}} \in L^{1}(\mathbb{R})$. Therefore, we have obtained the solvability of the equation for this case, and we can give its explicit solution.

As about the case $\lambda=0$, we can prove without difficulty that $\mathcal{F}_{\alpha}[f] / \mathcal{F}_{\alpha}[k] \in L^{1}(\mathbb{R})$. For instance, if it is the Fourier case, then $F[f](s) / F[k](s)=2 a\left(a^{2}+s^{2}\right) e^{-\frac{1}{2} s^{2}}$. This function belongs to $L^{1}(\mathbb{R})$, and therefore it fulfills the condition in Theorems 8 .

Thus, in both cases all the conditions of Theorems 8 are fulfilled. Hence, the corresponding equation possesses a solution and we have the consequent explicit solution formula.

\section{ACKNOWLEDGEMENT}

P. K. Anh, P. T. Thao, and N. M. Tuan were partially supported by the Viet Nam National Foundation for Science and Technology Development (NAFOSTED). L. P. Castro was supported in part by FCT-Portuguese Foundation for Science and Technology through the Center for Research and Development in Mathematics and Applications (CIDMA) at Universidade de Aveiro, within UID/MAT/04106/2013.

\section{REFERENCES}

[1] T. Alieva, V. Lopez, F. Agullo-Lopez and L. B. Almeida, "The fractional Fourier transform in optical propagation problems", J. Mod. Opt. 41, 1037-1044 (1994).

[2] L. B. Almeida, "The fractional Fourier transform and time-frequency representation", IEEE Trans. Sig. Proc. 42, 3084-3091 (1994).

[3] L. B. Almeida, "Product and convolution theorems for the fractional Fourier transform", IEEE Signal Processing Letters 4(1), 15-17 (1997).

[4] P. K. Anh, L. P. Castro, P. T. Thao and N. M. Tuan, "Two new convolutions for the fractional Fourier transform", Wireless Personal Communications, 15pp, to appear.

[5] B. Barshan and B. Ayrulu, "Fractional Fourier transform pre-processing for neural networks and its application to object recognition", Neural Networks 15(1), 131-140 (2002).

[6] W. Beckner, "Inequalities in Fourier analysis", Annals of Math. 102, 159-182 (1975). 
[7] H. J. Brascamp and E. H. Lieb, "Best constants in Young's inequality, its converse and its generalization to more than three functions", Adv. Math. 20, 151-173 (1976).

[8] L. P. Castro, "A relation between convolution type operators on intervals in Sobolev spaces", Appl. Anal. 74, 393-412 (2000).

[9] L. P. Castro, "Regularity of convolution type operators with PC symbols in Bessel potential spaces over two finite intervals", Math. Nachr., 261-262, 23-36 (2003).

[10] L. P. Castro and F.-O. Speck, "Relations between convolution type operators on intervals and on the halfline”, Integral Equations Operator Theory 37, 169-207 (2000).

[11] L. P. Castro and D. Kapanadze, "The impedance boundary-value problem of diffraction by a strip", J. Math. Anal. Appl. 337, 1031-1040 (2008).

[12] L. P. Castro and B. Zhang, "Invertibility of convolution operators arising in problems of wave diffraction by a strip with reactance and Dirichlet conditions", Z. Anal. Anwend. 24, 545-560 (2005).

[13] E. U. Condon, "Immersion of the Fourier transform in a continuous group of functional transformations", Proc. National Academy Sciences 23, 158-164 (1937).

[14] B. Deng, R. Tao and Y. Wang, "Convolution theorems for the linear canonical transform and their applications”, Science in China Series F: Information Sciences 49(5), 592-603 (2006).

[15] I. Djurovic, S. Stankovic and I. Pitas, "Digital watermarking in the fractional Fourier transformation domain", Journal of Network and Computer Applications 24(2), 167-173 (2001).

[16] F. Garcia-Vicente, J. M. Delgado and C. Peraza, "Experimental determination of the convolution kernel for the study of the spatial response of a detector", Med. Phys. 25, 202-207 (1998).

[17] F. Garcia-Vicente, J. M. Delgado and C. Rodriguez, "Exact analytical solution of the convolution integral equation for a general profile fitting function and Gaussian detector kernel”, Phys. Med. and Biol. 45, 645650 (2000).

[18] B. T. Giang, N. V. Mau and N. M. Tuan, "Operational properties of two integral transforms of Fourier type and their convolutions", Integral Equations Operator Theory 65, 363-386 (2009).

[19] B. T. Giang, N. V. Mau and N. M. Tuan, "Convolutions for the Fourier transforms with geometric variables and applications", Math. Nachr. 283, 1758-1770 (2010).

[20] F. Hlawatsch and F. G. Bourdeaux-Bartels, "Linear and quadratic time-frequency signal representations", IEEE Signal Processing Mag. 9(2), 21-67 (1992).

[21] H. Kober, "Wurzeln aus der Hankel-und Fourier und anderen stetigen Transformationen", Quart. J. Math. Oxford Ser. 10, 45-49 (1939).

[22] A. C. McBride and F. H. Kerr, “On Namia’s fractional order Fourier transform”, IMA J. Appl. Math. 39, 159-175 (1987).

[23] D. Mendlovic and H. M. Ozaktas, "Fractional Fourier transforms and their optical implementation: I", J. Opt. Soc. Amer. A 10, 1875-1881 (1993).

[24] V. Namias, "The fractional Fourier transform and its application to quantum mechanics", J. Inst. Math. Appl. 25, 241-265 (1980).

[25] H. M. Ozaktas, O. Arıkan, M. A. Kutay and G. Bozdağ1, "Digital computation of the fractional Fourier transform”, IEEE Trans. Signal Processing 44, 2141-2150 (1996).

[26] H. M. Ozaktas, B. Barshan and D. Mendlovic, "Convolution and filtering in fractional Fourier domains", Optical Review 1(1), 15-16 (1994).

[27] H. M. Ozaktas and D. Mendlovic, "Fractional Fourier transforms and their optical implementation: II", J. Opt. Soc. Amer. A 10, 2522-2531 (1993).

[28] H. M. Ozaktas, Z. Zalevsky and M. A. Kutay, The Fractional Fourier Transform with Applications in Optics and Signal Processing (John Wilay and Sons, New York, 2001).

[29] W. Rudin, Functional Analysis, second ed. (McGraw-Hill, New York, 1991).

[30] Q. Ran, H. Zhang, J. Zhang, L. Tang and J. Ma, "Deficiencies of the cryptography based on multipleparameter fractional Fourier transform", Optics Letters 34(11), 1729-1731 (2009).

[31] A. K. Singh and R. Saxena, "On convolution and product theorems for FRFT", Wireless Personal Communications 65(1), 189-201 (2012).

[32] E. C. Titchmarsh, Introduction to the Theory of Fourier Integrals, third ed. (Chelsea Publishing Co., New York, 1986).

[33] D. Wei, Q. Ran, Y. Li, J. Ma and L. Tan, "A convolution and product theorem for the linear canonical transform", IEEE Signal Processing Letters 16(10), 853-856 (2009).

[34] N. Wiener, "Hermitian polynomials and Fourier analysis", J. Math. Phys. 8, 70-73 (1929).

[35] A. I. Zayed, "A convolution and product theorem for the fractional Fourier transform", IEEE Signal Processing Letters 5(4), 102-103 (1998). 